\title{
Another Proof of Hadamard's Determinantal Inequality
}

\author{
FINBARR HOLLAND
}

\begin{abstract}
We offer a new proof of Hadamard's celebrated inequality for determinants of positive matrices that is based on a simple identity, which may be of independent interest.
\end{abstract}

A hermitian $n \times n$ matrix $A$ is said to be positive, if, for all $n \times 1$ vectors $x, x^{*} A x>0$ unless $x$ is the zero vector. Thus, if $A$ is positive, all of its principal sub-matrices are also positive. Moreover, $A$ is positive if and only if the determinants of all these sub-matrices are positive. In particular, if $A=\left[a_{i j}\right]$ is positive, then all of its diagonal entries, $a_{11}, a_{22}, \ldots, a_{n n}$, and its determinant, $\operatorname{det} A$, are positive. These are well-known facts about positive matrices that can be found in most textbooks on Matrix Analysis, such as, for instance, [1] and [3].

In 1893, Hadamard [2] discovered a fundamental fact about positive matrices, viz., that, for such $A=\left[a_{i j}\right]$,

$$
\operatorname{det} A \leq a_{11} a_{22} \cdots a_{n n} \text {. }
$$

Our purpose here is to present another proof of Hadamard's inequality which is based on the following identity.

Lemma 1. Suppose $A$ is an $n \times n$ matrix, $\tilde{A}$ is its cofactor matrix, and $x, y$ are $n \times 1$ vectors. Then

$$
\operatorname{det} A-\operatorname{det}\left[\begin{array}{cc}
A & x \\
y^{t} & 1
\end{array}\right]=x^{t} \tilde{A} y \text {. }
$$

Proof. Identify $\mathbb{C}^{n}$ with the space of $n \times 1$ vectors with complex entries, and consider the bilinear form

$$
B(x, y)=\operatorname{det} A-\operatorname{det}\left[\begin{array}{cc}
A & x \\
y^{t} & 1
\end{array}\right], x, y \in \mathbb{C}^{n} .
$$


Denoting the usual orthonormal basis of $\mathbb{C}^{n}$ by $e_{1}, e_{2}, \ldots, e_{n}$, it's easy to see that

$$
B\left(e_{i}, e_{j}\right)=A_{i, j},
$$

the $i j$ th element in $\tilde{A}$. Hence, if

$$
x=\sum_{i=1}^{n} x_{i} e_{i}, y=\sum_{i=1}^{n} y_{i} e_{i} \in \mathbb{C}^{n},
$$

then, by bilinearity,

$$
\begin{aligned}
B(x, y) & =\sum_{i, j=1}^{n} x_{i} y_{j} B\left(e_{i}, e_{j}\right)=\sum_{i, j=1}^{n} x_{i} y_{j} A_{i j} \\
& =\sum_{i=1}^{n} x_{i} \sum_{j=1}^{n} A_{i j} y_{j}=x^{t} \tilde{A} y
\end{aligned}
$$

as stated.

As an easy consequence, we have:

Theorem 1. Suppose $A$ is an $n \times n$ positive matrix. Then

$$
\operatorname{det}\left[\begin{array}{cc}
A & x \\
x^{*} & 1
\end{array}\right] \leq \operatorname{det} A \quad\left(x \in \mathbb{C}^{n}\right),
$$

with equality if and only if $x=0$.

Proof. Since $A$ is invertible, and its inverse is also positive, it follows from the lemma that

$$
\operatorname{det} A-\operatorname{det}\left[\begin{array}{cc}
A & x \\
x^{*} & 1
\end{array}\right]=x^{*} \tilde{A} x=\operatorname{det} A x^{*} A^{-1} x \geq 0,
$$

and the inequality is strict unless $x$ is the zero vector. The result follows.

Corollary 1. Denoting by $A_{k}$ the sub-matrix of $A$ of order $k \times k$ that occupies the top left-hand corner of $A=\left[a_{i j}\right]$, then

$$
\operatorname{det} A \leq a_{n n} \operatorname{det} A_{n-1},
$$

and the inequality is strict unless all the entries in the last column of $A$, save the last one, are zero. 
Hadamard's classical inequality is an immediate consequence of this, viz.,

Theorem 2 (Hadamard). If $A=\left[a_{i j}\right]$ is an $n \times n$ positive matrix, then

$$
\operatorname{det} A \leq \prod_{i=1}^{n} a_{i i},
$$

with equality if and only if $A$ is a diagonal matrix.

Coupling this with the fact that the determinant of $A$ is the product of its eigenvalues, $\lambda_{1}, \lambda_{2}, \ldots, \lambda_{n}$, say, we can affirm that

$$
\prod_{i=1}^{n} \lambda_{i} \leq \prod_{i=1}^{n} a_{i i}
$$

with equality if and only if $A$ is a diagonal matrix. But, also, the sum of the eigenvalues of $A$ is its trace, i.e.,

$$
\sum_{i=1}^{n} \lambda_{i}=\sum_{i=1}^{n} a_{i i}
$$

In other words, denoting by $\sigma_{r}\left(x_{1}, x_{2}, \ldots, x_{n}\right)$ the $r$ th symmetric function of $n$ variables, $x_{1}, x_{2}, \ldots, x_{n}$, we have that

$$
\sigma_{r}\left(\lambda_{1}, \lambda_{2}, \ldots, \lambda_{n}\right) \leq \sigma_{r}\left(a_{11}, a_{22}, \ldots, a_{n n}\right),
$$

if $r=1$ or $r=n$. It's of interest to observe that this remains true if $1<r<n$. For completeness, we sketch a proof of this statement

Indeed, $\sigma_{r}\left(\lambda_{1}, \lambda_{2}, \ldots, \lambda_{n}\right)$ is the coefficient $a_{r}$ of $t^{n-r}$ in the polynomial

$$
\prod_{i=1}^{n}\left(t+\lambda_{i}\right)=\operatorname{det}(A+t I)
$$

But $a_{r}$ is equal to the sum of the determinants of all the $r \times r$ principal sub-matrices of $A$, which are also positive. Hence, applying Hadamard's result to each of them, we deduce that $a_{r} \leq$ $\sigma_{r}\left(a_{11}, a_{22}, \ldots, a_{n n}\right)$ as claimed. 


\section{REFERENCES}

[1] R. Bhatia, Matrix Analysis, Springer Graduate Texts in Mathematics, Vol. $169,1997$.

[2] J. Hadamard, Résolution d'une question relative aux déterminants, Bulletin des sciences math. (2), 17 (1893), 240-248.

[3] L. Mirsky, An Introduction to Linear Algebra, Oxford University Press, 1963.

Finbarr Holland,

Mathematics Department,

University College, Cork,

Cork, Ireland

f.holland@ucc.ie

Received on 31 May 200\%. 